\title{
SOME RESULTS ON INVARIANT THEORY
}

\author{
BY S. HELGASON ${ }^{1}$ \\ Communicated by Felix Browder, April 11, 1962
}

1. Symmetric invariants. Let $V$ be a finite-dimensional vector space over $R$. Each $X \in V$ gives rise (by parallel translation) to a vector field on $V$ which we consider as a differential operator $\partial(X)$ on $V$. The mapping $X \rightarrow \partial(X)$ extends to an isomorphism of the complex symmetric algebra $S(V)$ over $V$ onto the algebra of all differential operators on $V$ with constant complex coefficients. Let $G$ be a subgroup of the general linear group $G L(V)$. Let $I(V)$ denote the set of $G$-invariants in $S(V)$ and let $I_{+}(V)$ denote the set of $G$-invariants without constant term. The group $G$ acts on the dual space $V^{*}$ of $V$ by

$$
\left(g \cdot v^{*}\right)(v)=v^{*}\left(g^{-1} \cdot v\right), \quad g \in G, v \in V, v^{*} \in V^{*},
$$

and we can consider $S\left(V^{*}\right), I\left(V^{*}\right), I_{+}\left(V^{*}\right)$. An element $p \in S\left(V^{*}\right)$ (a polynomial function on $V$ ) is called $G$-harmonic if $\partial(J) p=0$ for each $J \in I_{+}(V)$. Let $H\left(V^{*}\right)$ denote the set of $G$-harmonic polynomial functions.

Let $V^{c}$ denote the complexification of $V$. Suppose $B$ is a nondegenerate symmetric bilinear form on $V^{c} \times V^{c}$. If $X \in V^{c}$ let $X^{*}$ denote the linear form $Y \rightarrow B(X, Y)$ on $V$. The mapping $X \rightarrow X^{*}$ extends to an isomorphism $P \rightarrow P^{*}$ of $S(V)$ onto $S\left(V^{*}\right)$. If $G$ leaves $B$ invariant then $I(V)^{*}=I\left(V^{*}\right)$.

We shall use the following notation: If $E$ and $F$ are linear subspaces of the associative algebra $A$ then $E F$ denotes the set of all sums $\sum_{i} e_{i} f_{i},\left(e_{i} \in E, f_{i} \in F\right)$.

THEOREM 1. Let $B$ be a nondegenerate symmetric bilinear form on $V \times V$ and let $G$ be a Lie subgroup of $G L(V)$ leaving $B$ invariant. Suppose that either (1) $G$ is compact and $B$ positive definite or (2) $G$ is connected and semisimple. Then

$$
S\left(V^{*}\right)=I\left(V^{*}\right) H\left(V^{*}\right) .
$$

The case of a compact $G$ was noted independently by B. Kostant. It is a simple consequence of the fact that under the standard strictly positive definite inner product on $S\left(V^{*}\right)$ (invariant under $G$ ), the space $H\left(V^{*}\right)$ is the orthogonal complement to the ideal in $S\left(V^{*}\right)$ generated by $I_{+}\left(V^{*}\right)$. For the noncompact case, let $g$ denote the complexification of the Lie algebra of $G$. It is not difficult to prove that

1 This work was supported by the National Science Foundation, NSF G-19684. 
each compact real form $\mathfrak{u}$ of $\mathfrak{g}$ leaves invariant a real form $W$ of $V^{c}$ on which $B$ is strictly positive definite. Now the compact case can be applied to the action of $\mathfrak{u}$ on $W$.

In the case when $G$ is the orthogonal group $O(n)$ acting on $V=R^{n}$ then $I\left(V^{*}\right)$ consists of all polynomials in $x_{1}^{2}+\cdots+x_{n}^{2}$ and $H\left(V^{*}\right)$ consists of all the ordinary harmonic polynomials. Theorem 1 reduces to the classical fact that each $p=p\left(x_{1}, \cdots, x_{n}\right)$ can be written $p=\sum_{k}\left(x^{2}+\cdots+x_{n}^{2}\right)^{k} h_{k}$ where each $h_{k}$ is harmonic. It is also known (compare Cartan [2, p. 285], Maass [9]) that $H\left(V^{*}\right)$ is in this case spanned by the polynomials $\left(a_{1} x_{1}+\cdots+a_{n} x_{n}\right)^{k}$ where $a_{1}, \cdots, a_{n}$ $\in C, a_{1}^{2}+\cdots+a_{n}^{2}=0$ and $k=0,1, \cdots$. The following generalization holds:

TheOREM 2. Let the assumptions be as in Theorem 1. Let $N_{G}$ denote the set of common zeros $\left(\right.$ in $\left.V^{c}\right)$ of the elements in $I_{+}\left(V^{*}\right)$. Then $H\left(V^{*}\right)$ is the direct sum

$$
H\left(V^{*}\right)=H_{1}\left(V^{*}\right)+H_{2}\left(V^{*}\right),
$$

where $H_{1}\left(V^{*}\right)$ is the vector space spanned by the polynomials $\left(X^{*}\right)^{k}$, $\left(k=0,1,2, \cdots, X \in N_{G}\right)$ and $H_{2}\left(V^{*}\right)$ is the set of G-harmonic polynomials which vanish identically on $N_{G}$.

For the case $G=O(n)$ it follows easily from Hilbert's Nullstellensatz that $H_{2}\left(V^{*}\right)=0$.

2. Exterior invariants. Let $\Lambda(V)$ and $\Lambda\left(V^{*}\right)$, respectively, denote the Grassmann algebras over the dual vector spaces $V$ and $V^{*}$. Each $X \in V$ induces an antiderivation $\delta(X)$ of $\Lambda\left(V^{*}\right)$ given by

$\delta(X) \cdot\left(x_{1} \wedge \cdots \wedge x_{n}\right)=\sum_{k=1}^{n}(-1)^{k+1} x_{k}(X)\left(x_{1} \wedge \cdots \wedge \hat{x}_{k} \wedge \cdots \wedge x_{n}\right)$

where $\hat{x}_{k}$ indicates omission of $x_{k}$. The mapping $X \rightarrow \delta(X)$ extends uniquely to an isomorphism of $\Lambda(V)$ into the algebra of all endomorphisms of $\Lambda\left(V^{*}\right)$. Let $G$ be any subgroup of $G L(V)$. Let $J(V)$ and $J\left(V^{*}\right)$ denote the set of $G$-invariants in $\Lambda(V)$ and $\Lambda\left(V^{*}\right)$, respectively, $J_{+}(V)$ and $J_{+}\left(V^{*}\right)$ the sets of invariants without constant term. An element $p \in \Lambda\left(V^{*}\right)$ is called $G$-primitive if $\delta(J) p=0$ for each $J \in J_{+}(V)$. Let $P\left(V^{*}\right)$ denote the set of $G$-primitive elements.

THEOREM 3. Let the assumptions be as in Theorem 1. Then

$$
\Lambda\left(V^{*}\right)=J\left(V^{*}\right) \wedge P\left(V^{*}\right) .
$$

Example. Let $E$ be an $n$-dimensional Hilbert space over $C$. Considering $E$ as a $2 n$-dimensional vector space $V$ over $R$ the unitary 
group $U(n)$ becomes a subgroup $G$ of the orthogonal group $O(2 n)$. Let $Z_{k}=X_{k}+i Y_{k}(1 \leqq k \leqq n)$ be an orthonormal basis of $E$ and let $x_{1}, y_{1}, \cdots, x_{n}, y_{n}$ be the basis of $V^{*}$ dual to the basis $X_{1}, Y_{1}, \cdots$, $X_{n}, Y_{n}$ of $V$. It is easy to show that the element

$$
u=\sum_{1}^{n} x_{k} \wedge y_{k} \quad\left(=\frac{i}{2} \sum_{1}^{n} z_{k} \wedge \bar{z}_{k}\right)
$$

and its powers form a basis of $J_{+}\left(V^{*}\right)$. In view of Theorem 3 each $v \in \Lambda\left(V^{*}\right)$ can therefore be written

$$
v=\sum_{k} u^{k} \wedge p_{k}
$$

where each $p_{k}$ satisfies $\delta(u) p_{k}=0$, (compare Weil [10, Théorème 3, p. 26]).

3. Invariants of Weyl groups. Let $\mathfrak{u}$ be an arbitrary semisimple Lie algebra over $R$ whose adjoint group $U$ is compact. Let $\theta$ be an arbitrary involutive automorphism of $\mathfrak{u}$ and let $\mathfrak{u}=\mathfrak{l}+\mathfrak{p}$ be the decomposition of $\mathfrak{u}$ into eigenspaces of $\theta$ for the eigenvalue +1 and -1 respectively. Let $K$ denote the analytic subgroup of $U$ corresponding to $\mathfrak{l}$. Let $\mathfrak{h}_{\mathfrak{p}}$ be a maximal abelian subspace of $\mathfrak{p}$ and extend $\mathfrak{h}_{\mathfrak{p}}$ to a maximal abelian subalgebra $\mathfrak{h}$ of $\mathfrak{u}$. The Weyl group of $\mathfrak{h}$ is defined as the group of linear transformations of $\mathfrak{h}$ induced by the set of elements in $U$ which leave $\mathfrak{h}$ invariant; the Weyl group of $\mathfrak{h}_{p}$ is defined as the group of linear transformations of $\mathfrak{h}_{\mathrm{p}}$ induced by the set of elements in $K$ which leave $\mathfrak{h}_{\mathfrak{p}}$ invariant. Let these groups be denoted by $W(\mathfrak{h})$ and $W\left(\mathfrak{h}_{\mathfrak{p}}\right)$ and let $I\left(\mathfrak{h}^{*}\right)$ and $I\left(\mathfrak{h}_{\mathrm{p}}^{*}\right)$ denote the corresponding sets of invariant polynomial functions. It is known that $W\left(\mathfrak{h}_{\mathfrak{p}}\right)$ can be described as the group of linear transformations of $\mathfrak{h}_{\mathfrak{p}}$ induced by those members of $W(\mathfrak{h})$ which leave $\mathfrak{h}_{\mathfrak{p}}$ invariant. Consequently, if the restriction to $\mathfrak{h}_{\mathfrak{p}}$ of a function $f$ on $\mathfrak{h}$ is denoted by $\bar{f}$, the mapping $f \rightarrow \bar{f}$ maps $I\left(\mathfrak{h}^{*}\right)$ into $I\left(\mathfrak{h}_{p}^{*}\right)$.

Theorem 4. (i) Suppose $\mathfrak{u}$ is a classical compact simple Lie algebra and $\theta$ any involutive automorphism of $\mathfrak{u}$. Then the restriction mapping $f \rightarrow \bar{f}$ maps $I\left(\mathfrak{h}^{*}\right)$ onto $I\left(\mathfrak{h}_{p}^{*}\right)$.

(ii) Part (i) does not hold in general for the exceptional simple Lie algebras $\mathfrak{u}=\mathfrak{e}_{6}, \mathfrak{e}_{7}, \mathfrak{e}_{8}$.

(iii) Let $Q\left(\mathfrak{h}^{*}\right)$ and $Q\left(\mathfrak{h}_{p}^{*}\right)$, respectively, denote the set of invariant rational functions on $\mathfrak{h}$ and $\mathfrak{h}_{\mathfrak{p}}$. Under the restriction mapping $f \rightarrow \bar{f}$, $Q\left(\mathfrak{h}^{*}\right)$ is mapped onto $Q\left(\mathfrak{h}_{p}^{*}\right)$.

Remarks. As $\mathfrak{u}$ and $\theta$ are arbitrary, $\mathfrak{t}+i \mathfrak{p}$ is the most general semisimple Lie algebra over $R$. Parts (i) and (ii) above therefore express 
a property which is shared by all classical simple Lie algebras over $R$, yet fails to hold for all simple Lie algebras over $R$. Part (i) is proved by verification using Cartan's classification [1] of the root structures of $U$ and of the symmetric space $U / K$. Since the groups $W\left(\mathfrak{h}_{\mathfrak{p}}\right)$ and $W(\mathfrak{h})$ are finite groups generated by reflections, $I\left(\mathfrak{h}_{p}^{*}\right)$ and $I\left(\mathfrak{h}^{*}\right)$ are polynomial rings, (Chevalley [4]). The degrees of the generators can be readily determined from known facts. It is then found that if the space $U / K$ is $E_{6} / F_{4}, E_{7} /\left(E_{6} \times T\right)$ or $E_{8} /\left(E_{7} \times S U(2)\right)$, the ring $I\left(\mathfrak{h}_{\mathrm{p}}^{*}\right)$ contains a homogeneous element of degree 3,4 , and 6 , respectively, which cannot be obtained from $I\left(\mathfrak{h}^{*}\right)$ by restriction. Part (iii) had been proved independently by Harish-Chandra.

4. Fundamental functions on quadrics. Let $G$ be a topological group, $H$ a closed subgroup, $G / H$ the set of left cosets $g H$ with the natural topology. If $f$ is a complex-valued continuous function on $G / H$ and $x \in G$ then $f^{x}$ denotes the function on $G / H$ given by $f^{x}(g H)$ $=f(x g H)(g \in G)$. The function $f$ is called fundamental (Cartan [3, p. 218]) if the vector space $V_{f}$ over $C$ spanned by the functions $f^{x}(x \in G)$ is finite-dimensional.

Consider the quadric $C_{p, q} \subset R^{p+q}$ given by the equation

$$
Q(X) \equiv x_{1}^{2}+\cdots+x_{p}^{2}-x_{p+1}^{2}-\cdots-x_{p+q}^{2}=1, \quad(p \geqq 1, q \geqq 0) .
$$

Let $\boldsymbol{O}(p, q)$ denote the group of linear transformations of $\boldsymbol{R}^{p+q}$ leaving $Q$ invariant. The group $O(p, q)$ acts transitively on $C_{p, q}$ and the subgroup leaving $(1,0, \cdots, 0)$ fixed is isomorphic to $O(p-1, q)$ so we make the identification

$$
C_{p, q}=O(p, q) / O(p-1, q) .
$$

It is obvious that if $P=P\left(x_{1}, \cdots, x_{p+q}\right)$ is a polynomial then the restriction of $P$ to $C_{p, q}$ is a fundamental function. On the other hand we have

Theorem 5. Let $f$ be a fundamental function on $C_{p, q}$. Assume $(p, q) \neq(1,1)$. Then there exists a polynomial $P=P\left(x_{1}, \cdots, x_{p+q}\right)$ such that

$$
f=P \quad \text { on } C_{p, q} \text {. }
$$

REMARKs. 1. The special case $q=0$ (for which $O(p, q)$ is compact) was already proved by Hecke [6] and Cartan [3].

2. If $p=1$, the denominator in (1) is compact and by use of a compact real form of the complexification of the Lie algebra of $\boldsymbol{O}(1, q)$ this case can be reduced to the case 1 . This procedure fails 
for $q=1$ because $O(1,1)$ is not semisimple and the theorem fails to hold for $(p, q)=(1,1)$ as the example $f\left(x_{1}, x_{2}\right)=\cosh ^{-1}\left(\left|x_{1}\right|\right)$ shows. The case $(p, q)=(1,2)$ was settled by Loewner [8] using special features of the Lobatchefsky plane.

3. By a method of descent the remaining cases can be reduced to the case $x_{1}^{2}+x_{2}^{2}-x_{3}^{2}=1$ (which differs radically from the case $x_{1}^{2}-x_{2}^{2}$ $-x_{3}^{2}=1$ by the noncompactness of the isotropy group). Here one can make use of the special property of the identity component of the group $O(2,1)$, namely that every representation of it extends to a representation of the corresponding complex subgroup of $G L(3, C)$, (see Harish-Chandra [5]).

4. From Theorem 1 it is clear that the polynomial $P$ can be taken to be an $O(p, q)$-harmonic polynomial, that is a polynomial satisfying the equation

$$
\left(\frac{\partial^{2}}{\partial x_{1}^{2}}+\cdots+\frac{\partial^{2}}{\partial x_{p}^{2}}-\frac{\partial^{2}}{\partial x_{p}{ }^{2}+1}-\cdots-\frac{\partial^{2}}{\partial x_{p}^{2}+q}\right) P=0 .
$$

It follows that the function $f$ is necessarily a sum of eigenfunctions of the Laplace-Beltrami operator on $C_{p, q}$ (formed by means of the indefinite Riemannian metric on $\left.C_{p, q},[7]\right)$.

\section{BIBLIOGRAPHY}

1. E. Cartan, Sur certaines formes riemanniennes remarquables des géometries a groupe fondamental simple, Ann. Sci. École Norm. Sup. 44 (1927), 345-467. 1931.

2. - Lecons sur la géométrie projective complexe, Gauthier-Villars, Paris,

3. - Sur la determination d'un systeme orthogonal complet dans un espace de Riemann symétrique clos, Rend. Circ. Mat. Palermo 53 (1929), 217-252.

4. C. Chevalley, Invariants of finite groups generated by reflections, Amer. J. Math. 77 (1955), 778-782.

5. Harish-Chandra, Lie algebras and the Tannaka duality theorem, Ann. of Math. 51 (1950), 299-330.

6. E. Hecke, Über orthogonalinvariante Integralgleichungen, Math. Ann. 78 (1918), 398-404.

7. S. Helgason, Some remarks on the exponential mapping for an affine connection, Math. Scand. 9 (1961), 129-146.

8. C. Loewner, On some transformation semigroups invariant under Euclidean and non-Euclidean isometries, J. Math. Mech. 8(1950), 393-409.

9. H. Maass, Zur Theorie der harmonischen Formen, Math. Ann. 137 (1959) 142149.

10. A. Weil, Variêtés Kähleriennes, Hermann, Paris, 1958.

Massachusetts Institute of Technology 